\title{
BMJ Open Understanding perceptions and factors involved in do not resuscitate (DNR) decision making in the emergency department of a low-resource country: a mixed-methods study protocol
}

\author{
Kiran Azizi (D) , ${ }^{1}$ Shahan Waheed, ${ }^{1}$ Rubina Barolia, ${ }^{2}$ Naveed Ahmed, ${ }^{3}$ \\ Madiha Ismail ${ }^{3}$
}

To cite: Azizi K, Waheed S, Barolia R, et al. Understanding perceptions and factors involved in do not resuscitate (DNR) decision making in the emergency department of a low-resource country: a mixed-methods study protocol. BMJ Open 2020;10:e038915. doi:10.1136/ bmjopen-2020-038915

- Prepublication history for this paper is available online To view these files, please visit the journal online (http://dx.doi org/10.1136/bmjopen-2020038915).

Received 08 April 2020 Revised 20 July 2020 Accepted 31 July 2020

Check for updates

(c) Author(s) (or their employer(s)) 2020. Re-use permitted under CC BY-NC. No commercial re-use. See rights and permissions. Published by BMJ.

${ }^{1}$ Emergency Department, Aga Khan University Hospital, Karachi, Sindh, Pakistan ${ }^{2}$ School of Nursing, Aga Khan University Hospital, Karachi, Sindh, Pakistan

${ }^{3}$ Emergency Medicine, Aga Khan University Hospital, Karachi, Sindh, Pakistan

Correspondence to

Dr Kiran Azizi;

kiran.nazeem@gmail.com

\section{ABSTRACT}

Introduction Do not resuscitate (DNR) decision making is an integral component of emergency medicine practice. There is a paucity of data, protocols and guidelines regarding the perceptions and barriers that are involved in the interactions among healthcare professionals, patients and their caregivers regarding DNR decision making. The aim of this study is, therefore, to explore the perceptions and factors influencing DNR decision making in the emergency department and to evaluate the use of a context-based protocol for DNR decision making. Methods and analysis This will be a sequential mixed method study beginning with qualitative research involving in-depth interviews (IDIs) with patient family members and focus group discussion with healthcare professionals. The consensual qualitative approach will be used to perform a thematic analysis to the point of saturation. The expected outcome will be to identify key themes that suggest perceptions and factors involved in DNR decision making. After piloting, the derived protocol will then be used with a different group of individuals ( 150 healthcare professionals) who meet the eligibility criteria in a quantitative cross-sectional study with universal sampling. Data will be analysed using NVIVO in the qualitative phase and SPSS V.19 in the quantitative phase. The study findings will support the development of a standardised protocol for DNR decision making for healthcare professionals in the emergency department.

Ethics and dissemination The proposal was reviewed by the ethics review committee (ERC) of the institution (ERC \# 2020-1551-7193). The project is an institution SEED grant recipient PF139/0719. The results will be disseminated among participants, patient communities and healthcare professionals in the institution through seminars, presentations, brochures and emails. The findings will be published in a highly accessed peer-reviewed medical journal and will be presented at international conferences.

\section{BACKGROUND}

Emergency departments are potential venues for do not resuscitate (DNR) discussions due to the high expectations of patients and

\section{Strengths and limitations of this study}

- This study will support the development of the first standardised protocol for healthcare professionals in the emergency department for do not resuscitate (DNR) patients.

- Later, the findings can be used to establish guidelines for DNR patients in emergency departments in developing countries.

- A context-based protocol will be developed using mixed methods to gain an understanding of the DNR process through qualitative data, and the derived protocol will be tested quantitatively.

- Qualitative interviews of both patients and healthcare professionals will be conducted to gain an understanding of their perceptions.

- It is a single-centre study with a small sample size.

family members regarding the early provision of care and patient disposition. ${ }^{1}$ This discussion occurs when patients or family members decline cardiopulmonary resuscitation based on a consideration of the poor prognosis of the disease or likelihood of survival with a severely compromised quality of life. ${ }^{12}$ The dynamic and fast-paced environment in emergency departments leads to multiple challenges. ${ }^{3}$ Due to the critical presentation of the patient, it is paramount that the emergency physician initiates a discussion with the patient's family members to decide on the acceptable level of invasiveness in the treatment plan in a timely manner. There are social, clinical and economic implications of these decisions in the low-resource setting of developing countries, imposing additional burdens on the fragile healthcare system. ${ }^{56}$

DNR decisions have been useful for preventing unnecessary and unwanted invasive treatment at the end of life. To reach 
an agreement regarding a DNR order, the evaluation of ethical competencies plays a pivotal role in understanding and exploring the factors that influence DNR decision making. ${ }^{7}$ The literature suggests that a mandatory requirement of effective patient-centred healthcare is the involvement of the patient and the patient's family members in the decision-making process. ${ }^{78}$ The DNR order discussion and the level of acceptance of DNR orders vary among countries. The rates at which DNRs are signed by ED patients are estimated to be $25 \%$ and $27 \%$ in South Korea and USA, respectively. The factors influencing $\mathrm{DNR} /$ code discussions in various studies were advanced age, poor prognosis, multiple comorbidities, advanced cardiac, pulmonary, hepatic or renal diseases, longer length of stay in the intensive care unit, malignancy and female sex. ${ }^{910}$

Currently, a significant proportion of patients with critically ill and unsalvageable conditions participate in DNR discussions in our emergency departments, ${ }^{11-13}$ further burdening low-resource countries, such as Pakistan, where there are few emergency departments. There is poor communication among the patient, their surrogates and the treating physician. Additionally, it is a prevailing misconception in the community that ventilator support is painful and expensive, and there is no guarantee that the patient will recover completely. ${ }^{14-16}$ Therefore, the decision to sign a DNR order places immense pressure on the patient's family, treating physicians and nursing staff, who have joint responsibilities to help the patient and their families make the right decision. ${ }^{17} 18$ In Pakistan, due to a robust joint family system, such decisions are made by the family members, who may have limited decision-making capacity in such stressful circumstances. The factors, perspectives and barriers that patients, family, nurses and physicians take into consideration when making such decisions in the emergency department have not been previously studied. Moreover, there are no guidelines or protocols that can help patients, families and physicians make such decisions. ${ }^{18-20}$

\section{AIM}

The study aims to evaluate the perceptions and factors that influence the DNR decision-making of patients presenting to the emergency department in a lowresource setting and to generate a DNR decision-making protocol for healthcare professionals.

\section{Research questions}

Research question 1

What are the perceptions of patients, families and healthcare professionals regarding the decision to sign a DNR order in the emergency department?

\section{Research question 2}

What are the individual-level and system-level perceived factors that affect the DNR decision-making process in the emergency department?
Research question 3

How effective is the derived DNR protocol for healthcare professionals in the emergency department?

\section{Research objectives}

1. To explore the perceptions of patients, families, emergency physicians and nurses regarding the code discussion pertaining to DNR orders in the emergency department.

2. To identify the individual-level and system-level perceived factors affecting the DNR decision-making process in the emergency department and develop an instrument.

3. To evaluate the derived DNR decision-making protocol for healthcare professionals in the emergency room

\section{METHODS AND DESIGN}

\section{Study design and setting}

The study will be a sequential mixed method study undertaken in the Emergency Department of Aga Khan University Hospital (AKUH). The study will occur in three phases: the first two phases will be qualitative, followed by a quantitative phase. AKUH is a tertiary care centre located in Karachi, a megacity that comprises a hybrid population with diverse socioeconomic and ethnic backgrounds.

\section{Phases I and II: qualitative analysis}

The qualitative data will be collected to ascertain the perceptions and views of the participants. This phase consists of two parts. First, a qualitative guide will be used to conduct in-depth interviews (IDIs) with the family members (caregivers/decision makers) of patients (online supplemental 1) after obtaining consent. The guide for the focus group discussions (FGDs) will be devised based on the findings of the IDIs. In the second part of the qualitative phase, FGDs will be conducted with healthcare professionals after consent is obtained to generate a consensus on the flow of the DNR decisionmaking process in the emergency department.

\section{Perceived individual-level factors}

Individual-level factors are defined as factors that are specific to individuals based on their demographics, socioeconomic status, comorbidities or religious beliefs, all of which play integral roles in DNR decision making. These include the age and sex of the patient, the relationship of the person signing the DNR order to with the patient, the educational status of the person signing the DNR order, religious beliefs, financial situation, comorbidities, length of stay in the special care units/intensive care units during a previous admission and family members' preferences (online supplemental 1).

\section{Perceived system-level factors}

System-level factors are defined as factors that are related to the emergency department system, such as overcrowding, short durations of physician-patient interactions, fast-paced working environment, limited space 
for privacy, time constraints for explaining DNR orders, lack of proper place/room for such discussions in the hospital, care and attitude of the doctors after the DNR order is signed, care and attitude of the nurses after the DNR order is signed, emergency department diversion and the busyness of shifts (online supplemental 1).

Participants in IDIs will be purposively selected from the emergency department of the hospital from January to August 2020. The study involves the recruitment of family members visiting with the patients, preferably those involved in DNR order decision making. Research staff trained specifically for this study will identify the participants from the emergency department and obtain informed consent.

The same recruitment process will be followed to enrol healthcare professionals in the study. The participants in the FGDs will be a heterogeneous group which includes nurses and doctors involved in patient care with varied levels of experience in the emergency department. Each will have discussed DNRs at least once.

In this phase, the main focus is on the quality of information, rather than the sample size. Hence, the sample size is dependent on the purpose of the study. The University of California Los Angeles guidelines suggest that 10-20 interviews with key informants lead to saturation of the data in exploratory studies. ${ }^{15}$ Hence, all possible efforts will be made to ensure the maximum recruitment of participants.

Furthermore, the FGDs and IDIs will be moderated by the research team, who will be trained in the conduct of qualitative studies. The language will be either the local language (Urdu) or English, which will be chosen based on the preference of the participant. All FGDs and IDIs will be audio-recorded with prior permission for recording obtained from participants. The transcription of data will be performed in the same language, and any interviews conducted in Urdu will be later translated into English. Considering the sensitivity of the topic, if any emotional distress is observed during the IDIs, the interview will be immediately terminated and a counselling session will be conducted by the research team.

Qualitative content analysis of the transcripts will be performed using a consensual qualitative approach until the point of saturation. The sequential mixed-method study phases are shown in figure 1, flow diagram.

The data gathered from the IDIs and FGDs will be analysed and used to generate a questionnaire. The developed questionnaire will be assessed with the Content Validity Index (CVI). The CVI exercise will include two emergency department physicians, senior nurse practitioners, psychiatrists, statisticians and a qualitative study expert. Lincoln and Guba's criteria will be used to assure the quality of the qualitative data. Qualitative research with credibility, conformability, transferability and dependability is considered the gold standard. ${ }^{16}$ Credibility refers to the certainty of the data and its interpretation. ${ }^{16}$ To fulfil this requirement, a researcher trained in qualitative research with in-depth knowledge of the subject will be recruited. To ensure conformability, field notes will be taken, and an audio recording of each interview will be made. Transferability means that the results and findings of the study are transferable to similar settings, which depends purely on the design of the research and the selection of the sample. ${ }^{16}$ In this study, we intend to recruit the maximum number of participants possible to extract an adequate amount of information about the topic. The study findings will be reported using the consolidated criteria for reporting qualitative research.

\section{Sampling strategy}

A purposive sampling strategy will be used for the qualitative portion of the study.

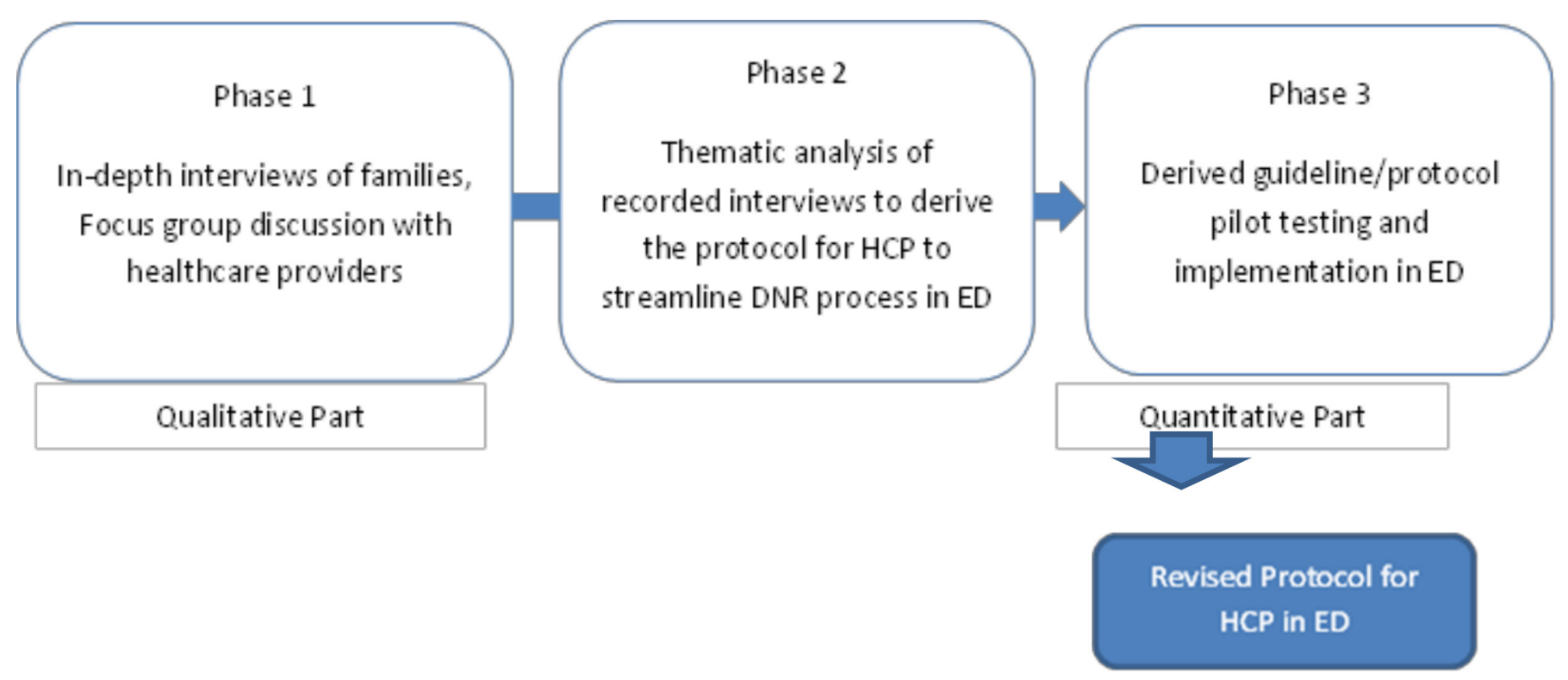

Figure 1 Flow chart: phases of this study. ED, emergency department; DNR, do not resuscitate; HCP, healthcare professionals. 


\section{Study population}

Patients, patients' families, emergency department physicians and nurses involved in discussions about DNR orders will constitute the study population.

\section{Eligibility criteria}

1. Adult patients aged 18 years and above involved in DNR discussions in the emergency department and who have the ability to give consent for participation will be recruited.

2. Patients' family members (decision-makers) who are involved in DNR decision making in the emergency department will be recruited.

3. Emergency department physicians and nurses (who have participated in at least one DNR discussion in clinical practice) will be recruited.

\section{Sample size for the qualitative analysis}

In qualitative research, the main focus is the quality of information obtained from the participants, rather than the size of the sample. Hence, the sample size is dependent on the purpose of the study and the saturation of the collected information. ${ }^{21}$ (Approximately 20-24 (10-12 family members, 10-12 healthcare professionals) IDIs and FGDs will be performed.

\section{Qualitative analysis}

Qualitative content analysis of the transcripts will be performed using a consensual qualitative approach to the point of saturation. The expected outcome of this thematic analysis will be to identify key themes that suggest the perceptions and factors involved in DNR decision making. This analytical approach involves several steps. The transcribed data will be thoroughly read by the two researchers separately to ensure that they comprehensively understand the meaning of the text. Then, the data will be labelled based on that understanding with codes. Furthermore, after reaching a consensus, the codes will be groups to form themes and subthemes.

\section{Phase III: quantitative analysis}

The derived protocol will be piloted and then used in a different group of individuals (healthcare professionals) who meet the eligibility criteria after they provide informed consent. The population that will be surveyed in the quantitative phase will be emergency department physicians and nurses who are taking care of patients or who have witnessed or participated in DNR discussions. The quantitative phase will have a cross-sectional design.

We will report our findings in the final integration phase of our research. First, the protocol will be piloted with healthcare professionals ( $10 \%$ of the sample). Then, the protocol will be used by healthcare professionals conducting DNR discussions with patients. Afterwards, they will be asked to provide feedback on the barriers faced, difficulties experienced in implementing the protocol, areas for improvement and the aspects they liked and disliked. For this purpose, 10 healthcare professionals and 5 family members involved in DNR decision making from the same sample will be approached to provide feedback. Approximately 70000 patients visit our emergency department, out of whom 7-14 patients per day are involved in DNR discussions; this is comparable to the data reported internationally. In our hospital emergency department, there are 150 healthcare professionals. We will employ a universal sampling method, and all healthcare workers will be involved in the study.

\section{Sampling strategy}

Purposive sampling will be used for the quantitative portion of the study.

\section{Eligibility criteria}

1. Adult patients aged 18 years and above with whom DNR discussions are held in the emergency department and who have the cognitive capacity to give consent will be recruited.

2. Patients' family members (decision-makers) who are involved in DNR decision making in the emergency department will be recruited.

\section{Sample size for the quantitative analysis}

In the AKUH emergency department, there are 150 healthcare professionals. Universal sampling will be used, and all healthcare workers will be included.

\section{Data analysis plan}

The data will be entered and analysed in SPSS V.19. In the descriptive analysis, continuous variables will be represented by the means and $\mathrm{SD}$, and categorical variables will be represented as the frequencies and percentages. Regression analysis will be applied for the quantitative phase of the study.

\section{Ethics considerations and consent to participation and dissemination}

The proposal has been approved by the Ethics Review Committee at Aga Khan University, Pakistan (ERC number 2020-1551-7193). This study has also received a seed grant from Aga Khan University (PF139/0719). The participants will be the family members, the nursing staff, and the emergency department physicians who participate in DNR decisions. Prior written consent will be obtained from all participants in the emergency department after the DNR decision is made. Privacy and confidentiality will be maintained, study participation will be voluntary, and there will be the option to opt out at any time during the study. The information and views collected will be kept confidential. The recorded interview tapes and transcribed data will be kept safely locked. No identifiers will be used in the study, and data will be retained for up to 7 years as per the AKU data retention policy. The research will be published in an international journal. The results will be disseminated within the institution through seminars, conferences and presentations during grand rounds. Additionally, the findings will be incorporated into the institutional guidelines. The results will also be incorporated into an informational brochure 
for the general public that will be placed in the patient waiting area and provided to patients and their families to help them make DNR decisions in the future. The outcomes of each data collection phase will be disseminated separately among the individuals involved in the study and other healthcare professionals, including the patient community, during weekly meetings and emails. The findings will be published in a highly accessed peerreviewed medical journal and will be presented at international conferences.

Acknowledgements The authors wish to acknowledge the contributions of Aga Khan University Hospital.

Contributors KA and SW performed all the core work of this project. RB is a qualitative expert and mentor. She has supervised the team at every step and will also participate in the data analysis. NA and MI helped to draft the protocol. All authors have read and approved the manuscript.

Funding This work was supported through the SEED grant from Aga Khan University PF139/0719. The funding source has no input in the design and will not make any contributions during the analysis and dissemination of the results.

Competing interests None declared.

Patient and public involvement Patients were not involved in the design and conception of this study. Refer to the 'Methods and design' section for further details.

Patient consent for publication Not required.

Provenance and peer review Not commissioned; externally peer reviewed.

Open access This is an open access article distributed in accordance with the Creative Commons Attribution Non Commercial (CC BY-NC 4.0) license, which permits others to distribute, remix, adapt, build upon this work non-commercially, and license their derivative works on different terms, provided the original work is properly cited, appropriate credit is given, any changes made indicated, and the use is non-commercial. See: http://creativecommons.org/licenses/by-nc/4.0/.

ORCID iD

Kiran Azizi http://orcid.org/0000-0001-6633-3084

\section{REFERENCES}

1 Pettersson M, Höglund AT, Hedström M. Perspectives on the DNR decision process: a survey of nurses and physicians in hematology and oncology. PLoS One 2018;13:e0206550.
2 Fan J-S, Huang H-H, Chen Y-C, et al. Emergency department DNR order in patients with spontaneous intracerebral hemorrhage. Am J Emerg Med 2017;35:1850-4.

3 Raftery KA. Emergency medicine in southern Pakistan. Ann Emerg Med 1996;27:79-83.

4 Hemphill JC, Newman J, Zhao S, et al. Hospital usage of early donot-resuscitate orders and outcome after intracerebral hemorrhage. Stroke 2004;35:1130-4.

5 Moazam F, Families MF. Families, patients, and physicians in medical decisionmaking: a Pakistani perspective. Hastings Cent Rep 2000;30:28-37.

6 Schneiderman LJ, Jecker NS, Jonsen AR. Medical futility: its meaning and ethical implications. Ann Intern Med 1990;112:949-54.

7 Plomer A. The law and ethics of medical research: international bioethics and human rights: Routledge-Cavendish 2013.

8 Keating NL, Landrum MB, Rogers SO, et al. Physician factors associated with discussions about end-of-life care. Cancer 2010;116:998-1006.

9 Searight HR, Gafford J. Cultural diversity at the end of life: issues and guidelines for family physicians. Am Fam Physician 2005;71:515-22.

10 Yaguchi A, Truog RD, Curtis JR, et al. International differences in end-of-life attitudes in the intensive care unit: results of a survey. Arch Intern Med 2005;165:1970-5.

11 Kardamanidis K, Lim K, Da Cunha C, et al. Hospital costs of older people in New South Wales in the last year of life. Med J Aust 2007;187:383-6.

12 Chalfin DB, Trzeciak S, Likourezos A, et al. Impact of delayed transfer of critically ill patients from the emergency department to the intensive care unit. Crit Care Med 2007;35:1477-83.

13 Berger JT, DeRenzo EG, Schwartz J. Surrogate decision making: reconciling ethical theory and clinical practice. Ann Intern Med 2008;149:48-53.

14 Jesieum M. Guidelines for conducting Key-Informants 2004.

15 Polit DF, Beck CT. Nursing research: principles and methods. Lippincott Williams \& Wilkins, 2004.

16 Siegrist V, Eken C, Nickel CH, et al. End-Of-Life decisions in emergency patients: prevalence, outcome and physician effect. QJM 2018;111:549-54.

17 Perkins GD, Fritz Z, Gavin DP. Time to change from do-notresuscitate orders to emergency care treatment plans. JAMA Netw Open 2019;2:e195170.

18 Wang A-Y, Ma H-P, Kao W-F, et al. Characteristics and outcomes of "Do Not Resuscitate" patients admitted to the emergency department-Intensive care unit. J Formos Med Assoc 2019;118:223-9.

19 Baker EFet al. Advance directives in the emergency department. JACEP Open 2020:1-6.

20 Immad SQ. DNAR decisions in Pakistan, middle East, and the UK: an emergency physician's perspective. South Asian Journal of Emergency Medicine 2018;2:1-5.

21 Saunders B, Sim J, Kingstone T, et al. Saturation in qualitative research: exploring its conceptualization and operationalization. Qual Quant 2018;52:1893-907. 